\title{
Prediction of treatment benefit in high-dimensional cox models via gene signatures in randomized clinical trials
}

\author{
Nils Ternès ${ }^{1,2^{*}}$, Federico Rotolo ${ }^{1,2}$, Georg Heinze ${ }^{3}$, Stefan Michiels ${ }^{1,2}$ \\ From 3rd International Clinical Trials Methodology Conference \\ Glasgow, UK. 16-17 November 2015
}

\section{Background}

Stratified medicine seeks to identify gene signatures predicting whether a patient will benefit from a treatment. We evaluated several approaches to identify such signatures using high-dimensional Cox models in randomized clinical trials (RCT).

\section{Methods}

We investigated four approaches: penalize biomarker main effects and biomarker-by-treatment interactions using a lasso penalty (full-lasso); control of main effects by principal components or ridge penalty, and lasso on interactions (sPCA+lasso or ridge+lasso); and 'modified covariates' in a penalized regression model (Tian et al. 2014). We performed simulations under null and alternative scenarios by varying the sample size $n$, number of biomarkers $H$, number of true main effects or treatment-modifiers, effect sizes and correlations. We proposed two novel measures of treatment effect prediction for gene signatures: a difference in C-indices and a Wald-based interaction statistic. We used gene expression data from a RCT of adjuvant chemotherapy in non-small cell lung cancer $(n=133)$ for illustration.

\section{Results}

When $n=500$ and $H=20$ or 100 , methods performed similarly in null scenarios apart from the full-lasso that gives poor results in presence of main effects only. In alternative scenarios: the ridge+lasso and the full-lasso predicted well the treatment benefit for future patients; the modified covariates approach performed poorly when also main effects were present. More extensive simulation results will be presented. In the lung cancer

${ }^{1}$ CESP, INSERM U1018, Paris-Sud Univ., Villejuif, France

Full list of author information is available at the end of the article trial, the full-lasso and the ridge+lasso selected a gene signature with four and seven treatment-modifiers.

\section{Conclusion}

Preliminary results suggest that ridge+lasso and full-lasso are promising approaches in high-dimensional Cox models to predict the treatment benefit.

\section{Authors' details}

${ }^{1}$ CESP, INSERM U1018, Paris-Sud Univ., Villejuif, France. ${ }^{2}$ Gustave Roussy, Service de biostatistique et d'épidémiologie, Villejuif, France. ${ }^{3}$ Center for Medical Statistics, Informatics and Intelligent Systems, Medical University of Vienna, Vienna, Austria.

Published: 16 November 2015

doi:10.1186/1745-6215-16-S2-086

Cite this article as: Ternès et al.: Prediction of treatment benefit in

high-dimensional cox models via gene signatures in randomized clinical trials. Trials 2015 16(Suppl 2):O86.

Submit your next manuscript to BioMed Central and take full advantage of:

- Convenient online submission

- Thorough peer review

- No space constraints or color figure charges

- Immediate publication on acceptance

- Inclusion in PubMed, CAS, Scopus and Google Scholar

- Research which is freely available for redistribution 